\title{
TNFSF10/TRAIL regulates human T4 effector memory lymphocyte radiosensitivity and predicts radiation-induced acute and subacute dermatitis
}

\author{
Jan Baijer ${ }^{1,2,3,4}$, Nathalie Déchamps ${ }^{1,2,3,4}$, Hervé Perdry ${ }^{5}$, Pablo Morales ${ }^{1}$, Sarah \\ Kerns ${ }^{6}$, Alexandre Vasilescu ${ }^{1}$, Sylvain Baulande ${ }^{7}$, David Azria ${ }^{8}$, Paul Henri \\ Roméo $^{1,2,3,4}$ and Annette Schmitz ${ }^{1}$ \\ ${ }^{1}$ CEA/DRF/IRCM/SCSR, Fontenay-aux-Roses Cedex, France \\ 2 Inserm U967, Fontenay-aux-Roses Cedex, France \\ 3 Université Paris-Diderot, Paris, France \\ ${ }^{4}$ Université Paris-Sud, Paris, France \\ ${ }^{5}$ Université Paris-Saclay, Univ. Paris-Sud, Inserm, CESP, Villejuif, France \\ ${ }^{6}$ University of Rochester Medical Center, Rochester, NY, USA \\ ${ }^{7}$ ICGex Next-Generation Sequencing Platform, Institut Curie, Paris, France \\ 8 Department of Radiation Oncology, ICM-Val d'Aurelle and INSERM U896, Institut de Recherche en Cancérologie de \\ Montpellier, Montpellier, France \\ Correspondence to: Paul Henri Roméo, email: paul-henri.romeo@cea.fr \\ Keywords: TRAIL, biomarker, lymphocyte, apoptosis, acute radiosensitivity \\ Received: November 13, 2015 Accepted: February 18, $2016 \quad$ Published: March 16, 2016
}

\section{ABSTRACT}

Sensitivity of T4 effector-memory (T4EM) Iymphocytes to radiation-induced apoptosis shows heritability compatible with a Mendelian mode of transmission. Using gene expression studies and flow cytometry, we show a higher TNF-Related Apoptosis Inducing Ligand (TRAIL/TNFSF10) mRNA level and a higher level of membrane bound TRAIL (MTRAIL) on radiosensitive compared to radioresistant T4EM lymphocytes. Functionally, we show that mTRAIL mediates a pro-apoptotic autocrine signaling after irradiation of T4EM lymphocytes linking mTRAIL expression to T4EM radiosensitivity. Using single marker and multimarker Family-Based Association Testing, we identified 3 SNPs in the TRAIL gene that are significantly associated with T4EM lymphocytes radiosensitivity. Among these 3 SNPs, two are also associated with acute and subacute dermatitis after radiotherapy in breast cancer indicating that T4EM lymphocytes radiosensitivity may be used to predict response to radiotherapy. Altogether, these results show that MTRAIL level regulates the response of T4EM lymphocytes to ionizing radiation and suggest that TRAIL/TNFSF10 genetic variants hold promise as markers of individual radiosensitivity.

\section{INTRODUCTION}

Inter-individual differences in radiosensitivity are described since the discovery of the effects of ionizing radiation on human, and are now associated with toxicity that patients treated with radiotherapy may experience. Surprisingly, except for very rare monogenic diseases such as homozygous carriers of a mutated ATM gene [1], the genetic basis of individual radiosensitivity is poorly characterized. Numerous studies indicated a correlation between cellular- and clinical radiosensitivity as exemplified by the association between low $\mathrm{CD}^{+}-$ lymphocyte apoptosis and radiation induced late toxicity [2]. However, no consensus exists on available biological tests that can be reliably used for prediction of early- and/ or late clinical adverse effects associated with radiotherapy $[3,4]$. This absence of predictive tests for individual radiosensitivity together with deleterious side effects 
observed in a minority of patients treated by radiotherapy led to limitation of the radiation dose used in radiotherapy. Because of the direct relationship between radiation dose and tumor control, this limitation reduces efficacy of radiotherapy in the majority of patients. Thus, beyond technical developments such as optimizing radiation delivery, improving radiotherapy outcome requires a better understanding of the underlying mechanisms of individual radiosensitivity [5] that will ultimately allow personalized radiotherapy.

We studied human T-lymphocytes radiation-induced apoptosis to characterize any genetic contribution to radiosensitivity. Radiation induced apoptosis of CD4positive T-lymphocytes lacking expression of both CD62L and CD45RA (T4EM lymphocytes) displayed significant heritability and transmission of this radiosensitivity phenotype within a cohort of large-kindred families was compatible with a simple Mendelian genetic model [6].

Genotype-phenotype correlations can be a consequence of genetically driven variation in gene expression that may be inherited [7]. Gene expression heritability within and across tissues revealed tissue- and/ or cell-specific heritability [8] and trait-associated loci are enriched for expression quantitative trait loci (eQTL; [9]). In radio-genetics, understanding how genotype affects gene expression is an important goal that was in particular studied through the search for genetic markers among genes whose expression is modulated in response to irradiation $[10,11]$. Most candidate gene approaches were centered on DNA damage, repair and signaling, but associations were not replicated, notably in the first genome-wide association studies to screen for SNPs in relation to radiotherapy toxicity and second malignant neoplasm $[12,13]$.

Here we show that the level of expression of TRAIL/TNFSF 10 is critically related to the previously defined cellular radiosensitivity phenotype. Functional studies showed that TRAIL blocking antibody, exogenous soluble TRAIL and soluble DR5 drastically reduce radiation-induced apoptosis and that membrane-bound TRAIL-mediated pro-apoptotic signaling is dependent on modulation of TRAIL shedding. Finally, we identified SNPs in the TRAIL/TNFSF10 gene that are associated with the cellular radiosensitivity phenotype. Interestingly, two of those SNPs were genetically associated with a subset of acute clinical radiosensitivity toxicities in a cohort of breast cancer patients. The identification of the role of TRAIL/TNFSF10 in susceptibility to radiation-induced apoptosis in human T4EM-lymphocytes suggests that the TRAIL-signaling pathway is associated with individual radiosensitivity and sheds new light on the role of TRAIL/ TNFSF 10 in the response of the immune system to radiation.

\section{RESULTS}

\section{TRAIL/TNFSF10 mRNA level correlates with T4EM lymphocytes radiosensitivity}

The sensitivity of subpopulations of human T-lymphocytes to ionizing radiation-induced apoptosis was studied eighteen hours after irradiation (0-2 Gy) of PBMC samples of healthy blood donors using the previously defined radiosensitivity assay based on immunophenotyping and AnnexinV-labeling. Whereas the CD62L-positive T lymphocytes did not undergo apoptosis (Supplementary Figure 1), a dose-dependent increase of apoptosis was evidenced in the CD62L-negative, T4EM lymphocytes (Figure 1A). Exponential regression coefficients of dose-survival curves were used to classify human PBMC samples according to the radiosensitivity of their T4EM lymphocytes and radiosensitive and radioresistant samples were defined at the two ends of the T4EM lymphocytes radiosensitivity distribution.

To identify genes differentially expressed, array-based expression profiling of flow sorted T4EM lymphocytes of four radiosensitive and four radioresistant day-fresh samples was performed (Figure 1B; black circles). Using a radiosensitive to radioresistant ratio of more than 2 (or less than 0.5 ), an expression level at least 3 times over background and $p<0.01$, we identified 31 genes expressed at a higher level (mean DF 2.40; 2.01 to 4.42 ), and 33 genes expressed at a lower level (mean DF $-2.67 ;-2.01$ to -7.48 ) in radiosensitive T4EM lymphocytes (Supplementary Table 1). Ontology analysis identified three pathways associated with radiosensitivity, cytokinecytokine receptor interaction (hsa04060), immune response (GO:0006955), and death (GO:0016265) (Supplementary Table 2). Among the genes up-regulated in radiosensitive T4EM lymphocytes, TRAIL/TNFSF10 displayed a 2.34-fold higher expression level, and was further studied. Quantitative PCR analysis of TRAIL/ TNFSF 10 mRNA expression levels in flow sorted T4EM lymphocytes of "sensitive", "median" and "resistant" samples (respectively illustrated by "red", "green" and "blue" dots in the distribution of radiosensitivity phenotypes in Figure 1B) showed that TRAIL/TNFSF10 mRNA level was 5.7-fold higher in radiosensitive compared to radioresistant T4EM lymphocytes, whereas TRAIL/TNFSF10 mRNA levels of T4EM lymphocytes with "median" radiosensitivity were intermediate (Figure 1C). Only 1 out of 45 studied samples showed discordant T4EM lymphocytes radiosensitivity and TRAIL/TNFSF 10 expression level.

To study the effects of irradiation on TRAIL and TRAIL-receptors mRNA levels in T4EM lymphocytes, we performed multiplexed determination of their mRNA levels on independent triplicate sorts from radiosensitive 
and radioresistant T4EM lymphocytes at 2, 4, 6 and 24 hours after 2 Gy irradiation. Expression of the decoy receptor TNFRSF10c/DcR1 was below detection limit (data not shown). No significant effect of irradiation on TRAIL/TNFSF10, on decoy receptor TNFRSF10d/DcR2 (not shown), and on pro-apoptotic receptor TNFRSF10al DR4 mRNA levels was found in radiosensitive and radioresistant T4EM lymphocytes (Figure 1D, left and middle panels). In contrast, in both radioresistant and radiosensitive T4EM lymphocytes, mRNA levels of the pro-apoptotic receptor TNFRSF10b/DR5 started to increase 4 hours after irradiation and remained high at 6 and 24 hours after irradiation (Figure 1D, right panels).

Altogether, these results indicate an association between TRAIL mRNA level and T4EM lymphocytes radiosensitivity and suggest a role for TNRSF10b/DR5 in T4EM lymphocytes radiation induced apoptosis.

\section{Membrane bound TRAIL (mTRAIL) mediates pro-apoptotic autocrine signaling in irradiated T4EM lymphocytes}

To show any involvement of TRAIL in T4EM lymphocytes radiosensitivity, we first analyzed TRAIL protein level in radiosensitive and radioresistant T4EM lymphocytes. TRAIL can be soluble (sTRAIL) or membrane-bound (mTRAIL) $[14,15]$. Using sandwich ELISA detection, we could not detect any expression of soluble TRAIL (sTRAIL) indicating that the concentration of sTRAIL in the media was less than $20 \mathrm{pg} / \mathrm{ml}$ of sTRAIL in the media. To determine the expression level of membrane bound TRAIL (mTRAIL), we used a monoclonal antibody panel comprising an anti-mTRAIL (CD253) antibody, and found a four-fold higher expression of mTRAIL on radiosensitive compared to radioresistant T4EM lymphocytes (Figure 2A and Supplementary Figure 2). Thus, radiosensitivity of T4EM lymphocytes is associated with expression level of mTRAIL.
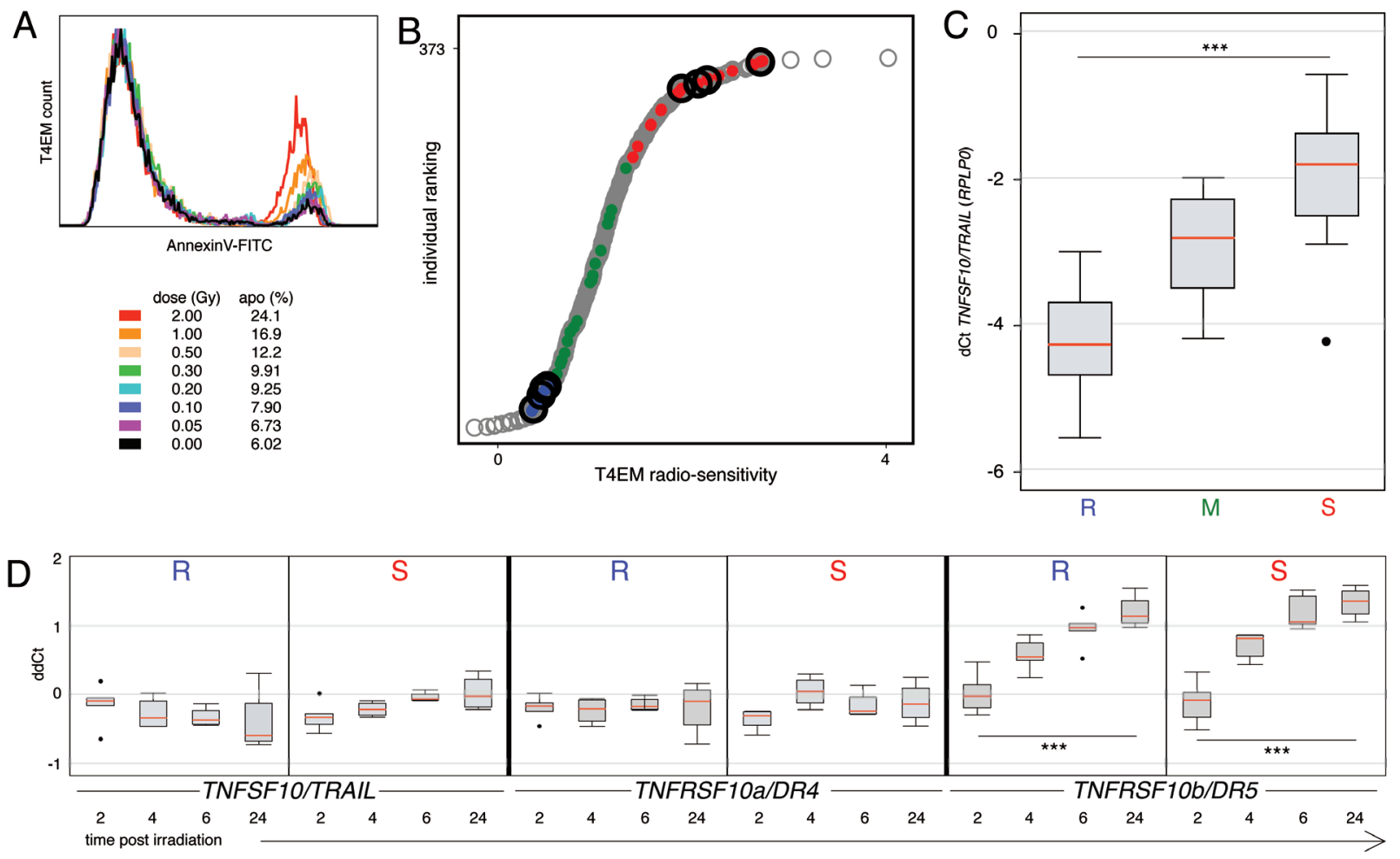

Figure 1: TRAIL/TNFSF10 mRNA level correlates with T4EM lymphocytes radiosensitivity. A. Dose dependent apoptosis of T4EM lymphocytes monitored by AnnexinV fluorescence intensity 18 hours after irradiation. B. Ranking of 373 individuals according to their T4EM lymphocytes radiosensitivity measured by the level of apoptosis (grey circles). T4EM lymphocytes from four "sensitive" and four "resistant" unrelated individuals (black circles) were selected for micro-array expression analysis. T4EM lymphocytes from fifteen "sensitive" (red), "median" (green) and "resistant" (blue) unrelated individuals were used for qPCR. C. Quantitative PCR analysis of expression level of TRAIL/TNFSF10 in sorted T4EM lymphocytes from 15 "resistant" (R), 15 "median" (M), and 15 "sensitive" unrelated individuals (S). Results are shown as box plots of $\mathrm{dCt}$ with respect to the internal reference gene (RPLP0) with median values (red) (*** $p$ $<10^{-3}$ ). D. Multiplexed qPCR analysis of TNFSF10/TRAIL (left panels), TNFRSF10a/DR4 (middle panels) and TNFRSF10b/DR5 (right panels) in sorted T4EM lymphocytes from 5 "resistant" (R) and 5 "sensitive" (S) unrelated individuals, 2, 4, 6, and 24 hours after irradiation at 2 Gy. Results are shown as box plots with median line (red) $\left(* * * p<10^{-3}\right)$ that represent, for each time point post-irradiation (hours), the difference in $\mathrm{dCt}(\mathrm{ddCt})$ between non-irradiated sample and the $\mathrm{dCt}$ of the test sample. 
To characterize the role of $\mathrm{mTRAIL}$ in radiationinduced apoptosis in T4EM, radiosensitive and radioresistant samples were irradiated in the presence of a soluble blocking antibody to human TRAIL (CD253; clone RIK-2). Apoptosis of T4EM lymphocytes in radioresistant samples was unaffected by the presence of the blocking antibody, whereas blocking of TRAIL resulted in strong inhibition of radiation-induced apoptosis of T4EM lymphocytes in radiosensitive samples, with levels of apoptosis in radiosensitive T4EM lymphocytes becoming similar to those observed in radioresistant T4EM lymphocytes, even after a 2.0 Gy irradiation (Figure 2B and 2C). Radiosensitive and radioresistant samples were then irradiated in the presence of recombinant human-soluble TRAIL (rh-sTRAIL), radiation induced apoptosis was inhibited in radiosensitive T4EM lymphocytes (Figure 2D and 2E). Inhibition of T4EM radiation-induced apoptosis was also observed when radiosensitive samples were irradiated in the presence of recombinant human soluble TRAIL DR5 Fc Chimera (rh-sDR5/Fc), a soluble form that antagonizes the proapoptotic receptor TNFRSF10b/DR5 [16]. This treatment decreased radiation-induced apoptosis of radiosensitive T4EM lymphocytes (Figure 2F). Finally, we studied any contribution of paracrine signaling in radiation-induced apoptosis of T4EM. At ten-fold higher cell concentrations, no increase in radiation-induced apoptosis could be detected, but on 9 "sensitive" samples, a ten-fold higher cell concentration resulted in 1.5-fold lower apoptosisinduction (Figure 2G).

Altogether, these results show that the radiationinduced apoptosis of T4EM lymphocytes is dependent on mTRAIL expression and suggest that mTRAIL mediates this radiation-induced apoptosis by an autocrine signaling involving the pro-apoptotic death receptors to TRAIL expressed by T4EM lymphocytes.

\section{1,10-Phenanthroline increases mTRAIL expression level and induces T4EM apoptosis}

Whereas the proteases involved in the shedding of TRAIL are not well characterized, metalloproteaseinhibitor 1,10-phenanthroline was shown to reduce the shedding of TRAIL [17]. Two hours after 1,10-phenanthroline treatment, mTRAIL expression level was increased in both radioresistant and radiosensitive T4EM lymphocytes (Figure 3A). Nevertheless, mTRAIL expression always remained more than 2 fold lower in 1,10-phenanthroline treated radioresistant T4EM lymphocytes than in untreated radiosensitive T4EM lymphocytes (Figure 3B).

In the absence of irradiation, 1,10-phenanthroline induced apoptosis of $\mathrm{B}$ and $\mathrm{T}$ lymphocytes (data not
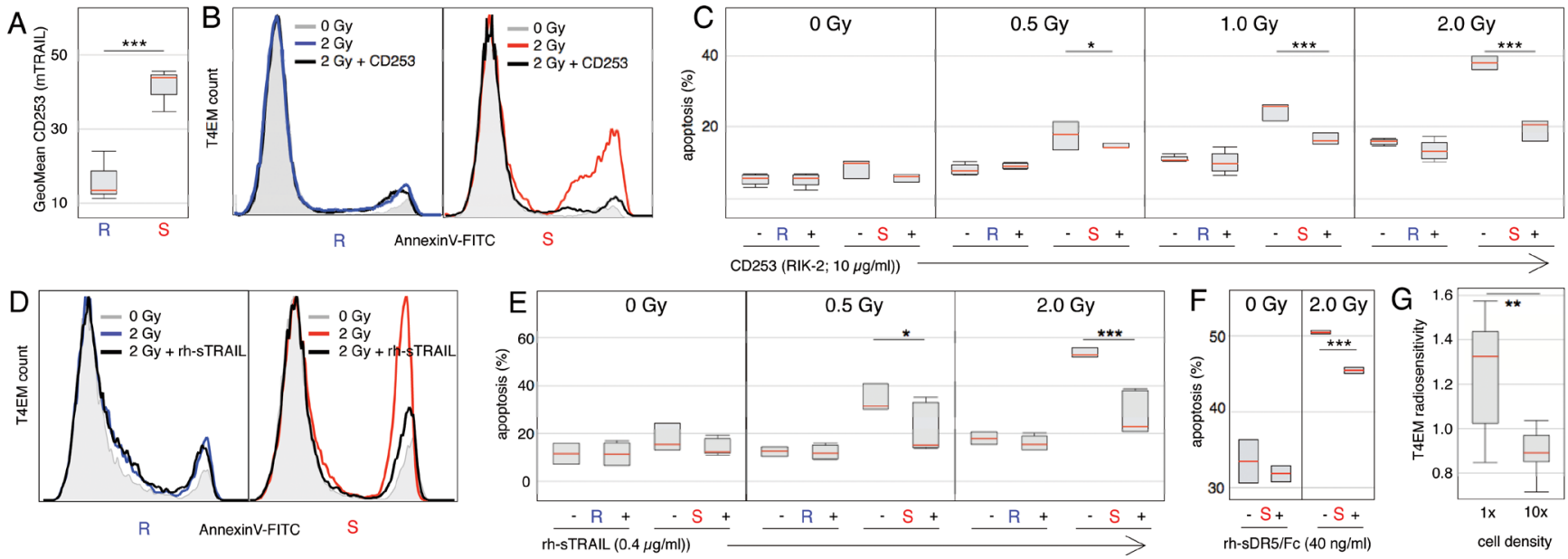

Figure 2: Membrane bound TRAIL (mTRAIL) mediates pro-apoptotic autocrine signaling in irradiated T4EM lymphocytes. A. mTRAIL level, quantified by flow cytometry, on resting T4EM lymphocytes from 4 "sensitive" (S) and 4 "resistant" (R) samples from unrelated individuals. Results are shown as box plots with median line (red) $\left(* * * p<10^{-3}\right)$. B. Apoptosis of T4EM lymphocytes monitored by AnnexinV fluorescence intensity of "resistant" (left panel) or "sensitive" (right panel) samples after 18 hours without irradiation (shaded grey), or after 2 Gy irradiation in the absence ("resistant" blue / "sensitive" red) or presence (black) of TRAIL blocking antibody (CD253). C. Percentage of apoptotic T4EM lymphocytes from 4 "sensitive" (S) and 4 "resistant" (R) samples, 18 hours after irradiation at $0,0.5,1$, and $2 \mathrm{~Gy}$, in the absence (-) or presence $(+)$ of TRAIL blocking antibody (CD253). Results are shown as box plots with median line (red) $\left({ }^{*} p<0.05,{ }^{* * *} p<10^{-3}\right)$. D. Apoptosis of T4EM lymphocytes monitored by AnnexinV fluorescence intensity of "resistant" (left panel) or "sensitive" (right panel) samples after 18 hours without irradiation (shaded grey), or after 2 Gy irradiation in the absence ("resistant" blue / "sensitive" red) or presence of $0.4 \mu \mathrm{g} / \mathrm{ml}$ rh-sTRAIL (black). E. Percentage of apoptotic T4EM lymphocytes from 4 "sensitive" (S) and 4 "resistant" (R) samples, 18 hours after irradiation at 0, 0.5, and 2 Gy, in the absence (-) or presence (+) of rh-sTRAIL $(0.4 \mu \mathrm{g} / \mathrm{ml})$. Results are shown as box plots with median line (red) $\left(* p<0.05, * * * p<10^{-3}\right)$. F. Percentage of apoptotic T4EM lymphocytes from 4 "sensitive" (S) samples, 18 hours after irradiation at 0 and 2 Gy, in the absence (-) or presence (+) of rh-sDR5/Fc (40 $\mathrm{ng} / \mathrm{ml})$. Results are shown as box plots with median line (red) $\left(* * * p<10^{-3}\right)$. G. Radiosensitivity of 9 samples from unrelated "sensitive" individuals after $2 \mathrm{~Gy}$ irradiation at cell concentrations of $10^{5} / \mathrm{ml}(1 \mathrm{X})$ and $10^{6} / \mathrm{ml}(10 \mathrm{X})\left(* * p<10^{-2}\right)$. 
Table 1: Single point association analysis (FBAT) between 15 frequent SNP of $T R A I L / T N F S F 10$ and radiosensitivity of T4EM

\begin{tabular}{|l|l|l|}
\hline Location & SNP & $\boldsymbol{p}$ \\
\hline Chr3:172,241,890 & rs12488654 & 0.08 \\
\hline Chr3:172,241,866 & rs365238 & 0.35 \\
\hline Chr3:172,241,759 & rs3136586 & 0.08 \\
\hline Chr3:172,240,999 & rs2270418 & 0.08 \\
\hline Chr3:172,229,871 & rs2241063 & 0.11 \\
\hline Chr3:172,228,544 & $\mathrm{rs} 3136597$ & 0.24 \\
\hline Chr3:172,227,199 & $\mathrm{rs3} 815496$ & 0.03 \\
\hline Chr3:172,224,771 & $\mathrm{rs} 17848019$ & 0.06 \\
\hline Chr3:172,224,303 & $\mathrm{rs} 1131532$ & 0.04 \\
\hline Chr3:172,224,075 & $\mathrm{rs} 1131535$ & 0.05 \\
\hline Chr3:172,223,926 & $\mathrm{rs} 1131542$ & 0.06 \\
\hline Chr3:172,223,690 & $\mathrm{rs} 1131568$ & 0.09 \\
\hline Chr3:172,223,627 & $\mathrm{rs} 1131579$ & 0.11 \\
\hline Chr3:172,223,620 & $\mathrm{rs} 1131580$ & 0.09 \\
\hline Chr3:172,223,376 & $\mathrm{rs} 11720451$ & 0.09 \\
\hline & & \\
\hline
\end{tabular}

shown). Specifically, T4EM lymphocytes underwent apoptosis 2 hours after treatment (Figure 3C). This apoptosis was mTRAIL dependent as it was inhibited when 1,10 phenanthroline treatment was performed in the presence of rh-sTRAIL (Figure 3D). Finally, using exponential regression coefficients of dose-survival curves on cryopreserved samples or fresh PBMC samples exposed to 1,10 phenanthroline or to ionizing radiation, we showed that radiosensitivity and sensitivity to 1,10 -phenanthroline were positively correlated (Figure 3D).

\section{Association of TRAIL/TNFSF10 SNPs with radiosensitivity and radio-induced acute and subacute dermatitis}

To investigate any genetic association between the TRAIL/TNFSF10 locus and radiosensitivity of T4EM lymphocytes, coding- and flanking regions of the TRAIL/TNFSF10 gene that contain SNPs that might be associated to- or act as eQTLs, were genotyped. A $4.2 \mathrm{~kb}$ region encompassing the TRAIL/TNFSF10 gene from 373 individuals previously characterized for T4EM lymphocytes radiosensitivity and subjected to heritability and segregation analysis [6] was sequenced. Supplementary Table 3 summarized results for the 36 polymorphic markers identified, with their frequencies and positions in the studied population. Single marker association analysis between the 15 identified frequent variants (MAF $>5 \%$ ) and T4EM radiosensitivity by Family-Based Association Testing (FBAT) showed a significant association for 3 SNPs; rs3815496 $(p=0.03$; intron 3, MAF $=0.28), \operatorname{rs} 1131532(p=0.04$; exon 5, MAF $=0.28)$, and $r s 1131535(p=0.05 ; 3$ ' UTR exon 5, MAF $=0.38)$. SNPs rs1131532 and rs3815496 were in strong linkage disequilibrium, and in partial linkage with rs1131535. Several other SNPs in the sequenced region were found to be marginally but significantly associated (Table 1). Multi-Marker association testing between the 15 most frequent SNPs of the TNFSF10/TRAIL gene and T4EM lymphocytes radiosensitivity was shown significant by the Family based MM association test (FBAT-MM) ( $p$ $=0.02)$ and by Fisher product $(p=0.04)$, demonstrating a genetic contribution to the T4EM lymphocytes radiosensitivity in or close to the TRAIL/TNFSF10 gene. The analysis of a selection of 126 unrelated individuals, using T4EM lymphocytes radiosensitivity median values by genotype at rs3815496 identified A as the risk allele for high levels of radiation-induced apoptosis in T4EM (Figure 4).

Association between the identified TRAIL/TNFSF10 SNPs and $\mathrm{CD}^{+}$lymphocyte apoptosis as well as with 
A

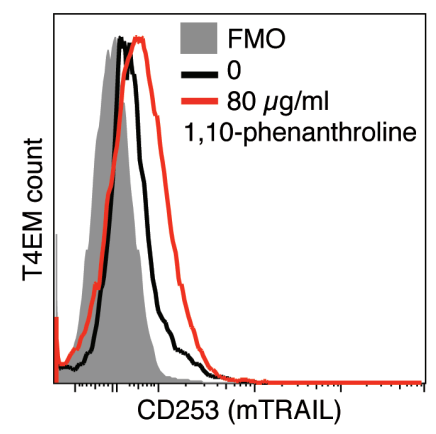

C

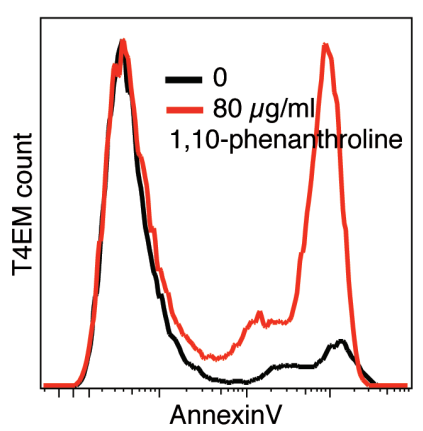

B

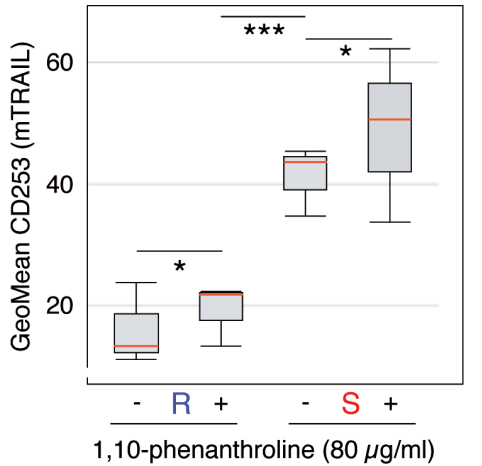

D

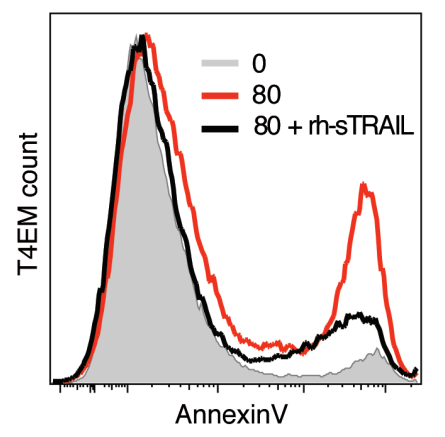

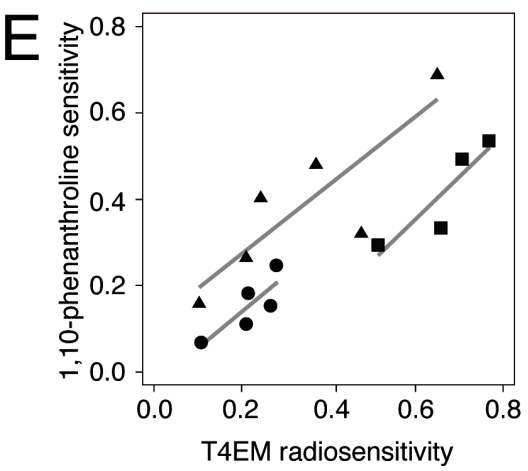

Figure 3: 1,10-Phenanthroline increases mTRAIL expression level and induces T4EM lymphocytes apoptosis. A. mTRAIL level, quantified by flow cytometry, on T4EM lymphocytes of a "sensitive" sample after 2 hours of treatment without (black) and with $80 \mu \mathrm{g} / \mathrm{ml}$ 1,10-phenanthroline (red). Corresponding FMO control histogram is shaded grey. B. mTRAIL expression on T4EM lymphocytes from 4 "sensitive" (S) and 4 "resistant" (R) samples two hours after treatment with $80 \mu \mathrm{g} / \mathrm{ml}$ of 1,10-phenanthroline. Results are shown as box plots with median line (red) $\left({ }^{*} p<0.05\right.$ and $\left.{ }^{* * *} p<10^{-3}\right)$. C. Apoptosis of T4EM lymphocytes, quantified by AnnexinV fluorescence, in a "sensitive" sample after two hours without (black) and with $80 \mu \mathrm{g} / \mathrm{ml} \mathrm{1,10-phenanthroline} \mathrm{(red).} \mathrm{D.} \mathrm{Apoptosis} \mathrm{of} \mathrm{T4EM}$ lymphocytes, quantified by AnnexinV fluorescence, after 2 hours without (grey) or with $80 \mu \mathrm{g} / \mathrm{ml}$ 1,10-phenanthroline in the absence (red) or in the presence of $4 \mu \mathrm{g} / \mathrm{ml} \mathrm{rh-sTRAIL} \mathrm{(black).} \mathrm{E.} \mathrm{Scatter} \mathrm{plot} \mathrm{of} \mathrm{T4EM} \mathrm{lymphocyte} \mathrm{radiosensitivity} \mathrm{(abscissa)} \mathrm{versus} \mathrm{T4EM} \mathrm{lymphocyte}$ sensitivity to 1,10-phenanthroline (ordinate). Three independent experiments were performed using 4 (cryopreserved; $\square), 5$ (fresh; •), and 6 (cryopreserved; -) samples. Regression lines were calculated for each experiment.

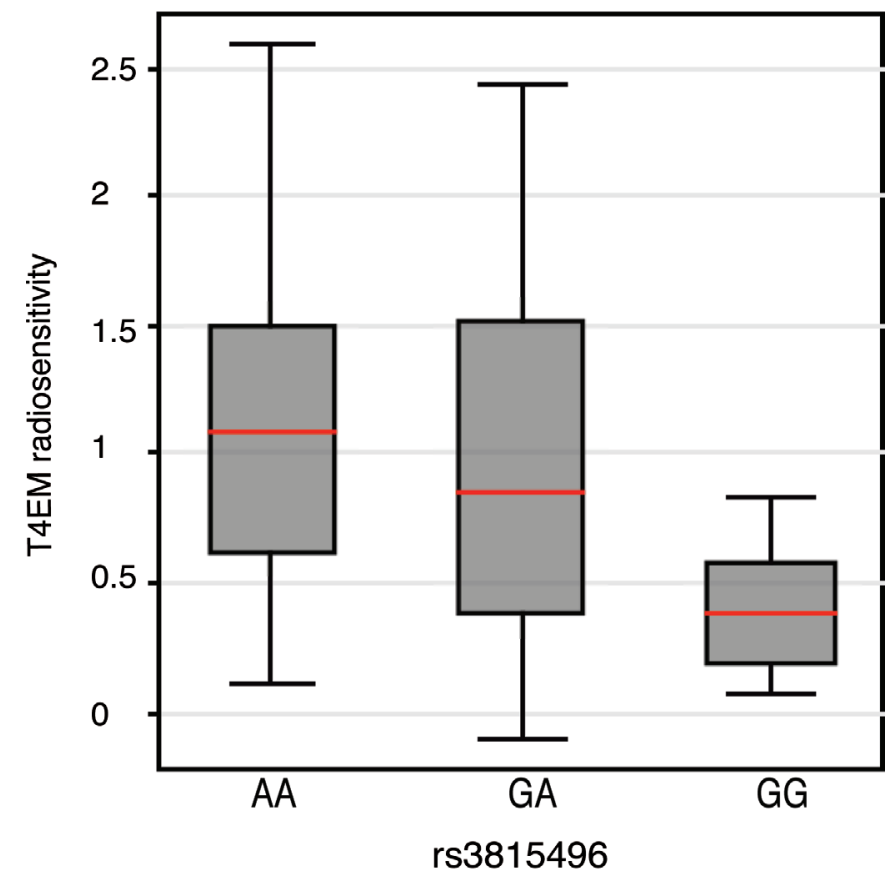

Figure 4: T4EM lymphocyte radiosensitivity monitored by rs3815496 genotype. Association between T4EM lymphocyte radiosensitivity and genotype at the rs3815496 SNP of the TRAIL/TNFSF10-gene. Results are shown as box plots with median line (red) for 126 unrelated individuals by genotype at rs3815496; AA $(n=65)$, GA $(n=55)$, and GG $(n=6)$. 
Table 2: Association testing between TRAIL/TNFSF10 SNP and CD8+ lymphocyte apoptosis and radiotherapyinduced skin reaction

\begin{tabular}{|c|c|c|c|}
\hline Endpoint & $\begin{array}{l}\text { rs1131535 } \\
(\text { effect allele }=\mathrm{A}, \\
\mathrm{MAF}=0.44)\end{array}$ & $\begin{array}{l}\text { rs1131532 } \\
(\text { effect allele }=C, \\
\text { MAF }=0.28)\end{array}$ & $\begin{array}{l}\text { rs3815496 } \\
(\text { effect allele }=\mathrm{A}, \\
\text { MAF }=\mathbf{0 . 2 8})\end{array}$ \\
\hline CD8+ lymphocyte apoptosis & $\begin{array}{l}1.84(-0.24,3.93) \\
p=0.086\end{array}$ & $\begin{array}{l}2.09(-0.09,4.27) \\
p=0.063\end{array}$ & $\begin{array}{l}2.08(-0.10,4.26) \\
p=0.064\end{array}$ \\
\hline $\begin{array}{l}\text { Acute } \& \text { subacute dermatitis } \\
(10 \text { cases, } 103 \text { controls })\end{array}$ & $\begin{array}{l}1.58(0.63,3.96) \\
p=0.336\end{array}$ & $\begin{array}{l}3.90(0.88,17.30) \\
p=0.045\end{array}$ & $\begin{array}{l}3.88(0.87,17.22) \\
p=0.047\end{array}$ \\
\hline $\begin{array}{l}\text { Acute erythema } \\
\text { ( } 44 \text { cases, } 69 \text { controls) }\end{array}$ & $\begin{array}{l}0.78(0.45,1.34) \\
p=0.364\end{array}$ & $\begin{array}{l}0.69(0.39,1.25) \\
p=0.252\end{array}$ & $\begin{array}{l}0.69(0.38,1.24) \\
p=0.244\end{array}$ \\
\hline $\begin{array}{l}\text { Acute hyperpigmentation } \\
\text { ( } 7 \text { cases, } 106 \text { controls) }\end{array}$ & $\begin{array}{l}1.71(0.57,5.09) \\
p=0.338\end{array}$ & $\begin{array}{l}1.49(0.40,5.52) \\
p=0.560\end{array}$ & $\begin{array}{l}1.48(0.40,5.50) \\
p=0.566\end{array}$ \\
\hline $\begin{array}{l}\text { Late fibrosis } \\
\text { ( } 28 \text { cases, } 83 \text { controls) }\end{array}$ & $\begin{array}{l}1.10(0.84,1.43) \\
p=0.486\end{array}$ & $\begin{array}{l}1.00(0.76,1.31) \\
p=0.987\end{array}$ & $\begin{array}{l}1.00(0.76,1.31) \\
p=0.975\end{array}$ \\
\hline $\begin{array}{l}\text { Late telangiectasia } \\
\text { (3 cases, } 108 \text { controls) }\end{array}$ & $\begin{array}{l}6.61(0.76,57.56) \\
p=0.045\end{array}$ & $\begin{array}{l}2.02(0.23,17.67) \\
p=0.517\end{array}$ & $\begin{array}{l}2.01(0.23,17.59) \\
p=0.521\end{array}$ \\
\hline
\end{tabular}

Association (SNPTEST) was evaluated in a set of 113 genotyped breast cancer patients, included in the Co-Ho-RT study.

clinical radiosensitivity endpoints was studied in a set of 113 genotyped breast cancer patients, included in the Co-Ho-RT study [18] (Table 2). Blood samples from these patients are characterized for radiation-induced $\mathrm{CD}^{+}$T-lymphocyte apoptosis, according to Ozsahin [2]. Genetic association study indicated a trend towards association of the three SNPs, rs3815496 (MAF = $0.28, p=0.06)$, rs1131532 $(\mathrm{MAF}=0.28, p=0.06)$, and rs1131535 (MAF $=0.44, p=0.09)$, with radiationinduced $\mathrm{CD} 8^{+} \mathrm{T}$-lymphocyte apoptosis. Finally, acute and subacute dermatitis (10 cases), acute erythema (44 cases), acute hyperpigmentation ( 7 cases), late fibrosis ( 28 cases), and late telangiectasia ( 3 cases) were evaluated for association with these three TRAIL/TNFSF10 SNPs. None of these three SNPs was associated with late fibrosis, acute erythema, or acute hyperpigmentation. An association was shown with late telangiectasia but, due to insufficient number of cases, this result was not considered. Interestingly, for acute and subacute dermatitis, a strong association was identified with $\operatorname{rs} 3815496(p=0.047$, effect allele $=$ A, OR: $3.88,95 \%$ c.i: $0.87-17.22)$, and with rs $1131532(p=0.045$, effect allele $=$ C, OR $3.90,95 \%$ c.i: 0.88-17.30), but not with rs1131535 (Table 2).

Altogether, these results show a genetic association between the TRAIL/TNFSF10 locus and radiosensitivity of T4EM lymphocytes and suggest that TRAIL/TNFSF10 genetic variants might be used as markers of individual radiosensitivity

\section{DISCUSSION}

Advances in radiotherapy treatment suffer from the lack of validated tests and genetic markers that can be used to predict individual radiosensitivity. Here we show that the expression level of TRAIL/TNFSF10 in resting T4EM lymphocytes identifies individuals with a high, TRAIL dependent, apoptotic response to ionizing radiation of T4EM lymphocytes.

The TNFRSF10b/DR5 receptor regulates radiationinduced apoptosis in mice and in breast cancer cell lines [19-21]. We now show its increased expression in T4EM lymphocytes after irradiation, suggesting that this receptor might play a broader role in radiationinduced apoptosis. Whereas in TRAIL sensitive cells, adding recombinant human soluble TRAIL (rh-sTRAIL) results in apoptosis [22], we show an inhibiting effect of rh-sTRAIL and of TRAIL blocking antibody on the radiation-induced apoptosis of T4EM lymphocytes. Together with the apoptosis-reducing effect observed at higher cell concentrations and the increased expression of membrane-bound (mTRAIL) in radiosensitive T4EM lymphocytes, these results indicate that MTRAIL but not sTRAIL expression on T4EM regulates their radiationinduced apoptosis. Finally, as DR5 but not mTRAIL expression increased after irradiation, these results suggest a paracrine, mTRAIL dependent, activation of mTRAIL/ DR5 signaling after irradiation of T4EM lymphocytes, and 
pinpoint mTRAIL and TRAIL/TNFSF10 expression level as markers of T4EM radiosensitivity.

After 1,10-phenanthroline treatment, the increased level of mTRAIL in T4EM lymphocytes showed the effects of metalloproteases on TRAIL shedding [17] in these cells. Interestingly, mTRAIL expression level in radioresistant T4EM lymphocytes treated with 1,10-phenanthroline was still more than two times lower than in untreated radiosensitive T4EM lymphocytes. Therefore, differences in mTRAIL shedding between resistant and sensitive individuals do not account for differential mTRAIL protein level, suggesting that TRAIL/ TNFSF 10 mRNA level may regulate mTRAIL expression in T4EM lymphocytes.

Measurements of radiosensitivity using only cell based assays have faced uncontrolled experimental variability [23]. Here we show an association between T4EM lymphocytes radiosensitivity and three SNPs within the TRAIL/TNFSF10-gene. Interestingly, these same SNPs were associated with risk and/or treatment outcome in ovarian and breast cancer [24-26]. Further investigation is now needed to understand if and/or how these genetic variants influence TRAIL/TNFSF10 mRNA level.

$\mathrm{CD}^{+}$lymphocyte apoptosis was shown to predict radiation-induced late toxicity [2] and this correlation was recently extended using a prospective multicenter study [27]. Although we did not detect a strong association between the three TRAIL/TNFSF10 SNPs characterized in this study and $\mathrm{CD} 8^{+}$lymphocyte apoptosis, a trend towards such an association was evidenced indicating the need for further association studies using other cohorts.

No association between the TRAIL/TNFSF 10 SNPs and late fibrosis could be detected. However, the importance of TRAIL in the clinical response to radiation and in its physiopathology was suggested by the association between two of the three reported TRAIL/TNFSF10 SNPs and acute and subacute radioinduced dermatitis. Acute and subacute dermatitis are a subset of radiotherapy-induced skin reactions (RISR) that are characterized by acute skin injury associated with inflammation, and are graded in the CTCAE system. These dermatitides can also occur after coronary angiography or embolization procedures [28]. Thus, the SNPs characterized in this study might be useful both in radiotherapy and interventional procedures.

Altogether our study highlights a role of TRAIL in T4EM-lymphocyte radiation induced apoptosis and the association of TRAIL/TNFSF10 SNPs with subtypes of radio-induced dermatitis. International consortium research that will provide the power of GWAS will now enable to appreciate the relative contribution of the identified TRAIL/TNFSF10 SNPs to radiotherapy toxicity $[29,30]$.

\section{MATERIALS AND METHODS}

\section{Healthy donors and samples}

All samples were prepared from venous blood drawn from healthy normal volunteers between 9:00 am and 11:00 am. Their inclusion was preceded by a medical interview and informed written consent was obtained from all donors, in accordance with local ethics guidelines at Fondation Jean Dausset-CEPH or Etablissement Français du Sang, Hôpital Saint Louis, Paris.

\section{Samples}

Peripheral blood mononuclear cells (PBMC) were prepared from fresh (within 6h, average 180 min) anticoagulated blood samples, by standardized procedures, as previously described [31]. Briefly, $30 \mathrm{ml}$ of two-fold diluted blood were underlayered with $10 \mathrm{ml}$ of Histopaque ${ }^{\circledR}-1077$ (Sigma-Aldrich), and centrifuged at 2,000g for 20 minutes. The collected interlayer was washed twice with $40 \mathrm{ml}$ of RPMI1640/15\% Fetal Calf Serum (FCS), and used as further described, or collected in FCS/10\% DMSO for cryopreservation. Fractions of $1 \mathrm{ml}$ each, corresponding to $4 \mathrm{ml}$ of blood, were immediately frozen, using controlled temperature decrease, and stored in liquid nitrogen. Thawing of cells was performed by rapid transfer of cryotubes to $56{ }^{\circ} \mathrm{C}$ waterbath $(45 \mathrm{sec})$, addition of $1 \mathrm{ml}$ of FCS and transfer of contents to $12 \mathrm{ml}$ of RPMI $/ 15 \%$ FCS. Thawed cells were washed twice, to be finally resuspended in the appropriate volume of culture medium (RPMI1640/15\% FCS).

DNA extractions were performed from PBMC by classical phenol/chlorophorm procedures. Yield and purity were assessed spectrophotometrically at 260 and $280 \mathrm{~nm}$ (Thermo Scientific NanoDrop ${ }^{\text {TM }}$ 1000). Familial relations were recorded from oral interview and HLA-A sequencing was used to exclude from family-based association analysis those samples presenting incompatibilities with announced familial interrelations.

\section{Monoclonal antibodies and proteins}

All monoclonal antibodies were used at saturating conditions, as determined by titrations. Antihuman $\mathrm{CD}$ antibodies came from Becton Dickinson ImmunoCytometry Systems (CD62L-PE, CD62LPECy5.1, CD14-PECy5.1, CD-235a-PECy5.1, CD27PECy5.1, CD4-PECy7, CD19-PECy7, CD45RA-APC, CD8-APCH7), from Beckman Coulter (CD3-PETxR) from Biolegend (CD253/TRAIL-PE, CD62L-BV421, CD3-BV605), or from R\&D Systems (CD262/DR5APC). Recombinant human soluble TRAIL (rh-sTRAIL; 
Cat\# 375-TL), anti-TRAIL cytotoxicity blocking antibody (CD253; clone RIK-2) and recombinant human soluble DR5 Fc chimera (rh-sDR5/Fc; Cat\#631-T2) were from R\&D Systems, AnnexinV-FITC from Becton Dickinson, and 1,10-phenanthroline from Sigma-Aldrich.

\section{Apoptosis induction}

Cell suspensions at approximately $5 \times 10^{5}$ cells $/ \mathrm{ml}$ (or at cell densities specified in the results section) in RPMI1640/15\% FCS, were irradiated at a dose rate of approximately $0.5 \mathrm{~Gy} / \mathrm{min}$ with a ${ }^{137} \mathrm{Cs}$-source (IBL637; Cis-Bio international/Scherring, Saclay, France), and were incubated in the presence of indicated concentrations of rhsTRAIL, CD253, rh-sDR5/Fc, or of 1,10-phenanthroline (stock solution of $200 \mathrm{mM}$ in DMSO). All experiments described were performed with the same batch of heat inactivated FCS, previously tested for compatibility with our experimental procedures. Incubations of 18 hours or less as specified in results section were at $37{ }^{\circ} \mathrm{C}$ and $5 \%$ $\mathrm{CO} 2$.

\section{Cell labeling for lymphocyte subset identification and apoptosis quantification}

Treated cells were collected and washed with AnnexinV buffer (140mM NaCl, 5mM CaCl2, 10mM HEPES; ph7.4), and stained with $2.5 \mu$ l AnnexinV-FITC in combination with fluorochrome-conjugated CD markers in $100 \mathrm{ul}$ for $30 \mathrm{~min}$. For cell sorting for gene expression analysis, Annexin V positive cells were excluded, and CD3 labeling was omitted to avoid TCR-mediated activation signaling. Furthermore, CD14 and CD235a were included to exclude monocytes and reticulocytes susceptible to contaminate the sort gates. CD27 was included for sorts of cryopreserved samples to avoid contamination of sort gates by central memory lymphocytes that transiently lost CD62L through sample manipulation procedures. For CD253 (mTRAIL) quantification, a compact panel designed to limit the need for compensation including AnnexinV, CD253, CD3, CD4, and CD62L was used.

\section{Flow cytometry}

Cells were washed in AnnexinV-buffer and just prior flow cytometry, $50 \mu \mathrm{L}$ of AnnexinV-buffer containing 0.2 $\mu \mathrm{g} / \mathrm{mL}$ Hoechst 33258 (Molecular Probes, Eugene, OR), was added for dead cell exclusion. Samples were analyzed using a three-laser CyAn LX system, (DakoCytomation, Fort Collins, CO) or a 5-laser SORP LSRII (Becton Dickinson). Cell sorting was performed using a custombuilt five-laser InFlux system (Becton Dickinson). All filters were from Chroma Technologies Inc., Brattleboro, Vermont, and all fluorescence signals were log-amplified, and stored in listmode. CompBeads (Becton Dickinson) were used to assist in determination of spill-over in every individual experiment (CD3-FITC was used instead of AnnexinV-FITC) and Fluorescence Minus One (FMO) controls were used where needed, in particular for mTRAIL quantifications (SortWare; Becton Dickinson or Flowjo version 9.6.4 ; Treestar, Ashland, OR). All sorts were performed in single droplet count mode, with an extended coincidence mask of one-and-a-half droplet. Doublet exclusion was performed by gating on the triggering parameter (FSC) versus time of flight.

\section{Data analysis}

Data was analyzed using FlowJo. Listmode file analysis proceeded through the exclusion of nonlymphocyte events, predominantly monocytes, by using a large scattergate, shaped to include apoptotic lymphocytes (lower FSC, higher SSC). Subsequently, dead cells were excluded on the basis of HO33258 fluorescence. $\mathrm{T}$ Lymphocytes were identified on the basis of positive PETxR (CD3) fluorescence. A PECy7 (CD4) versus APCH7 (CD8) histogram allowed identification of CD4-positive T lymphocytes (T4) and CD8-positive T lymphocytes (T8). An APC (CD45RA) versus $\mathrm{PE}$ (CD62L) histogram discriminated between naive (CD62L+CD45RA+), central memory (CM; CD62L+CD45RA-), effector memory (EM; CD62LCD45RA-), and terminal effector (TE; CD62LCD45RA+) T4 and T8. The proportion of apoptotic cells was determined by application of an identical gate on a bivariate plot of FSC versus FITC fluorescence (AnnexinV) to all of the identified subpopulations. Doseeffect curves were generated using at least four doses, and used for the quantitative evaluation of 1,10-phenanthroline sensitivity and radiation sensitivity, as previously described [31].

\section{Microarray cDNA hybridization analysis}

Cells of interest, recovered by centrifugation directly after sort, were lysed by RLT buffer (Qiagen) and cryopreserved until RNA extraction. Total RNA was extracted from a minimum of 300,000 cells using the RNeasy Plus Micro Kit (Qiagen). RNA samples were resuspended in RNase-free water and stored at $-80^{\circ} \mathrm{C}$. RNA yield and purity were assessed spectrophotometrically at 260 and $280 \mathrm{~nm}$ (Thermo Scientific NanoDrop ${ }^{\mathrm{TM}}$ 1000). RNA integrity was evaluated using a Bioanalyzer 2100 (Agilent). Microarray experiments and part of data analysis were performed by PartnerChip (Evry, France) following the Affymetrix-recommended procedures. Target was prepared from 4 sensitive and 4 resistant individuals. Targets were pooled after individual quality control in equal proportions and hybridized on a HU133 
2.0 plus array $(54,645$ probe sets) according to the Affymetrix Two-Cycle technical protocol. Fluorescent images were detected in a GeneChip Scanner 3000 (Affymetrix). Expression data and raw expression data (CEL files) were generated using GCOS software (Affymetrix). Quality control was assessed based on 3'/5' ratios of glyceraldehyde 3-phosphate dehydrogenase (GAPDH) and $\beta$-actin control probe sets.

\section{Real-time quantitative PCR gene expression analysis}

Triplicate sorts for qPCR gene expression studies were performed by direct deposition of cells in MicrAmp optical tubes (Applied Biosystems), containing $15 \mu \mathrm{l}$ of one step RT-qPCR mix. RT-qPCR pre-amplification mix contained Platinum Taq polymerase and SuperScript III reverse transcriptase (Invitrogen), a mixture of Taqman primer-probes, specific for the transcripts of interest (Supplementary Table 4; Applied Biosystems) at 0.2X concentration, CellsDirect One-Shot qRT-PCR buffer (Invitrogen), and SuperaseIn RNase inhibitor. Immediately following cell sorting, samples were thoroughly mixed, and reverse transcribed $\left(55^{\circ} \mathrm{C}\right.$ for $10 \mathrm{~min}, 50{ }^{\circ} \mathrm{C}$ for 50 min), and pre-amplified ( 14 cycles of PCR at $95^{\circ} \mathrm{C}$ for 15 sec and $62{ }^{\circ} \mathrm{C}$ for $45 \mathrm{sec}$ ). After pre-amplification, samples were diluted ten-fold, for gene-specific quantitative PCR (35 cycles of $95{ }^{\circ} \mathrm{C} / 15 \mathrm{sec}, 62^{\circ} \mathrm{C} / 45 \mathrm{sec}$ ) on a HT7900 (Applied Biosystems). All primers were standard Taqman assays, and reaction conditions were according to manufacturers indications. Results were expressed as the difference between the $\mathrm{Ct}$ of the internally amplified reference gene (VIC-labeled $R P L P 0, A C T B$, or $G A P D H$ ), and the FAM-labeled test gene, averaged over triplicate sorts, and termed dCT. Assay performance control on cryopreserved lymphocytes included mean $\mathrm{dCt}$ on different cryopreserved vials from same blood sample, mean $\mathrm{dCt}$ from independent blood samples from same individual. Samples from reference individuals were included in each experiment. Radiation induction of expression is expressed as the difference between $\mathrm{dCT}$ at dose $2.0 \mathrm{~Gy}$ and $\mathrm{dCt}$ at dose $0 \mathrm{~Gy}$, for each timepoint after irradiation, and was termed ddCT.

\section{DNA genotyping}

Primers were established in order to amplify by PCR the exon-containing DNA fragments and the promoters (Supplementary Table 5 and 6). The PCRs were performed in a $15 \mu \mathrm{L}$ reaction mixture containing $25 \mathrm{ng}$ of DNA. Sequencing reactions were performed according to the dye terminator method using an ABI PRISM ${ }^{\circledR} 3730 x 1$ DNA Analyzer (Applied Biosystems, Foster City, CA, USA). Alignment of experimental results, SNP discovery and genotyping were performed with the software Genalys.
The genomic sequence used for the alignment is TRAIL/ TNFSF10 (NC_000003.11). The genotypes for rs3136597 and rs2241063 were obtained by Taqman technology, assays C_27464917_20 and C__3260973_20 respectively (Applied Biosystems).

\section{Family based association testing of frequent variants (FBAT)}

The FBAT method and software [32, 33] was used to perform a single marker analysis between the frequent SNPs and the quantitative trait while taking into account the family structure (command fbat -o). Two methods have been used to obtain a $p$-value for the global association of TNFSF10 with the trait : a) The Fisher product [34] of the $p$-values obtained from the single point association analysis was taken, and its significance was assessed using 10,000 permutations, b) The FBAT Multi-Marker statistic $[35,36]$ was computed, and its significance was assessed using 10,000 permutations.

\section{Radiotherapy induced skin reaction association testing with TRAIL/TNFSF10 SNPs (SNPTEST)}

Acute and subacute dermatitis, acute erythema, acute hyperpigmentation and late fibrosis were assessed prospectively using the CTCAE v3 grading system in the CO-HO-RT trial [18]. Toxicity was treated as a binary outcome and was determined by taking the highest CTCAE grade occurrence during radiotherapy to 6 weeks after radiotherapy for acute toxicities and from 3 months after radiotherapy to the end of follow-up (minimum 2 years, maximum 8 years) for late toxicities. For each toxicity endpoint, patients were considered cases if they had CTCAE grade $\geq 2$ and controls if they had CTCAE grade $\leq 1$. The percentage of CD $8+$ lymphocytes undergoing radiation-induced apoptosis was treated as a continuous outcome.

Genomic DNA was isolated from blood, genotyped using a commercial genome-wide SNP array (Affymetrix SNP6.0, Affymetrix, San Diego, CA)]. Because rs1131532, and rs3815496 were not directly genotyped on the array, the surrounding region of chromosome 3 (position 100,000,001 to $200,000,000$, hg build 19) was imputed to the 1000 Genomes reference data using IMPUTE2 software (37]. Following imputation, the SNPs of interest were confirmed to be in Hardy-Weinberg equilibrium ( $p$-value $\left.>10^{-6}\right)$ and showed good imputation quality (info score $>0.3$ ). SNPTEST software [38] was used to analyze SNP-phenotype association with the frequentist test and expected method, which uses expected genotype counts (i.e. genotype dosages) obtained from imputation. An additive genetic inheritance model was assumed in all analyses. 


\section{Statistical analysis}

Statistical analyses and data presentation were performed with Stata (Stata Corp.,College Station, TX). In box plot representations of results, boxes extend from $25^{\text {th }}$ to $75^{\text {th }}$ percentiles, with whiskers indicating upper- and lower adjacent values (1.5 times interquartile range). Each box represents at least three independent experiments.

\section{ACKNOWLEDGMENTS AND GRANT SUPPORT}

Our work is dedicated to Prof. Gilles Thomas with whom we started this research. Warm thanks to Lynn Goldin for continued interest and precious input, to the "donneurs du panel Jean Dausset" cohort of healthy blood donors sampled at EFS Saint Louis, the "Génétique des Cellules Sanguines" (GCS) blood donors and biological sample collection and Clement Hoffman, author of the excellent databases serving this research. We acknowledge the Fondation Jean Dausset-CEPH for the many years of collaboration and for collection and administration of biological samples from the GCS protocol. This work was supported by CEA, Inserm, University Paris-Sud, University Paris-Diderot and EDF grants to AS and PHR.

\section{CONFLICTS OF INTEREST}

No potential conflicts of interest were disclosed by the authors.

\section{Editorial note}

This paper has been accepted based in part on peerreview conducted by another journal and the authors' response and revisions as well as expedited peer-review in Oncotarget.

\section{REFERENCES}

1. Natarajan AT, Meyers M. Chromosomal radiosensitivity of ataxia telangiectasia cells at different cell cycle stages. Hum Genet. 1979; 52:127-132.

2. Ozsahin M, Crompton N, Gourgou S, Kramar A, Li L, Shi Y, Sozzi, A, Zouhair, R Mirimanoff R, Azria D. CD4 and CD8 T-lymphocyte apoptosis can predict radiationinduced late toxicity: a prospective study in 399 patients. Clin Cancer Res. 2005; 11: 7426-7433.

3. Finnon P, Kabacik S, MacKay A, Raffy C, A'Hern R, Owen R, Badie C, Yarnold J, Bouffler S. Correlation of in vitro lymphocyte radiosensitivity and gene expression with late normal tissue reactions following curative radiotherapy for breast cancer. Radiother Oncol. 2012;105: 329-336.

4. Greve B, Bölling T, Amler SS, Rössler U, Gomolka
M, Mayer C, Popanda O, Dreffke K, Rickinger A, Tritz E, Eckardt-Schupp F, Sauerland C, Braselmann H, et al. Evaluation of different biomarkers to predict individual radiosensitivity in an inter-laboratory comparison-lessons for future studies. PLoS ONE. 2011; 7:e47185.

5. West CM, Barnett GC. Genetics and genomics of radiotherapy toxicity: towards prediction. Genome Med. 2011; 3: 52-66.

6. Schmitz A, Bayer J, Dechamps N, Goldin L, Thomas G. Heritability of susceptibility to ionizing radiation-induced apoptosis of human lymphocyte subpopulations. Int J Radiat Oncol Biol Phys. 2007; 68: 1169-1177.

7. Morley M, Molony C, Weber T, Devlin J, Ewens K, Spielman R, Cheung V. Genetic analysis of genome-wide variation in human gene expression. Nature. 2004; 430: 743-747.

8. Price AL, Helgason A, Thorleifsson G, McCarroll SA, Kong A, Stefansson K. Single-tissue and cross-tissue heritability of gene expression via identity-by-descent in related or unrelated individuals. PLoS Genet. 2011; 7:e1001317.

9. Nicolae DL, Gamazon E, Zhang W, Duan S, Dolan ME, Cox NJ. Trait-associated SNPs are more likely to be eQTLs: annotation to enhance discovery from GWAS. PLoS Genet. 2010; 6: e1000888.

10. Svensson JP, Stalpers LJA, Esveldt-van Lange REE, Franken NAP, Haveman J, Klein B, Turesson I, Vrieling $\mathrm{H}$, Giphart-Gassler M. Analysis of gene expression using gene sets discriminates cancer patients with and without late radiation toxicity. PLoS Med. 2006; 3: e422.

11. Smirnov DA, Morley M, Shin E, Spielman RS, Cheung VG. Genetic analysis of radiation-induced changes in human gene expression. Nature. 2009; 459: 587-591.

12. Best T, Li D, Skol AD, Kirchhoff T, Jackson SA, Yasui Y, Bhatia S, Strong LC, Domchek SM, Nathanson KL, Olopade OI, Huang RS, Mack TM, et el. Variants at 6q21 implicate PRDM1 in the etiology of therapy-induced second malignancies after Hodgkin's lymphoma. Nat Med. 2011; 17: 941-943.

13. Fachal L, Gómez-Caamaño A, Barnett GC, Peleteiro P, Carballo AM, Calvo-Crespo P, Kerns SL, Sánchez-García M, Lobato-Busto R, Dorling L, Elliott RM, Dearnaley DP, Sydes MR, et al. A three-stage genome-wide association study identifies a susceptibility locus for late radiotherapy toxicity at 2q24.1. Nat Genet. 2014; 46: 891-894.

14. Schneider P, Holler N, Bodmer JL, Hahne M, Frei K, Fontana A, Tschopp J. Conversion of membrane-bound Fas(CD95) ligand to its soluble form is associated with downregulation of its proapoptotic activity and loss of liver toxicity. J Exp Med. 1998; 187: 1205-1213.

15. Wajant H, Moosmayer D, Wüest T, Bartke T, Gerlach E, Schönherr U, Peters N, Scheurich P, Pfizenmaier K. Differential activation of TRAIL-R1 and -2 by soluble and membrane TRAIL allows selective surface antigen-directed 
activation of TRAIL-R2 by a soluble TRAIL derivative. Oncogene. 2001; 20: 4101-4106.

16. Walczak H, Degli-Esposti MA, Johnson RS, Smolak PJ, Waugh JY, Boiani N, Timour MS, Gerhart MJ, Schooley KA, Smith cA, Goodwin RG, Trauch CT. TRAIL-R2: a novel apoptosis-mediating receptor for TRAIL. EMBO J. 1997; 16: 5386-5397.

17. Yan L, Chaojun S, Chunyan W, Boquan J, Wei J, Duo Z. Metalloprotease inhibitors reducing the shedding of human TRAIL. CORD Conference Proceedings. 2011; 108-111.

18. Azria D, Belkacemi Y, Romieu G, Gourgou S, Gutowski M, Zaman K, Llacer Moscardo C, Lemanski C, Coelho M, Rosenstein B, Fenoglietto P, Crompton NEA, Ozsahin M. Concurrent or sequential adjuvant letrozole and radiotherapy after conservative surgery for early-stage breast cancer (CO-HO-RT): a phase 2 randomised trial. Lancet Oncol. 2010; 11: 258-265.

19. Coureuil M, Ugolin N, Tavernier M, Chevillard S, Barroca V, Fouchet P, Allemand I. Puma and Trail/Dr5 pathways control radiation-induced apoptosis in distinct populations of testicular progenitors. PLoS ONE. 2010; 5: e12134.

20. Luce A, Courtin A, Levalois C, Altmeyer-Morel S, Roméo P-H, Chevillard S, Lebeau J. Death receptor pathways mediate targeted and non-targeted effects of ionizing radiations in breast cancer cells. Carcinogenesis. 2009; 30: 432-439.

21. Finnberg N, Gruber JJ, Fei P, Rudolph D, Bric A, Kim S-H, Burns TF, Ajuha H Page R, Wu GS, Chen Y, McKenna WG, Bernhard E, et al. DR5 knockout mice are compromised in radiation-induced apoptosis. Mol Cell Biol. 2005; 25: 2000-2013.

22. Wiley SR, Schooley K, Smolak PJ, Din WS, Nicholl JK, Sutherland GR, Davis Smith T, Rauch C, Smith CA, Goodwin RG. Identification and characterization of a new member of the TNF family that induces apoptosis. Immunity. 1995; 3: 673-682.

23. Barnett GC, Kerns SL, Noble DJ, Dunning AM, West CML, Burnet NG. Incorporating Genetic Biomarkers into Predictive Models of Normal Tissue Toxicity. Clin Oncol (R Coll Radiol). 2015 ;27: 579-587.

24. Charbonneau B, Block MS, Bamlet WR, Vierkant RA, Kalli KR, Fogarty Z, Rider DN, Sellers TA, Tworoger SS, Poole E, Risch HA, Salvesen HB, Kiemeney LA. Risk of Ovarian Cancer and the NF- B Pathway: Genetic Association with IL1A and TNFSF10. Cancer Res. 2014; 74: 852-861.

25. FitzGerald LM, Kwon EM, Conomos MP, Kolb S, Holt SK, Levine D, Feng Z, Ostrander EA, Stanford JL. Genome-wide association study identifies a genetic variant associated with risk for more aggressive prostate cancer. Cancer Epidemiol Biomarkers Prev. 2011; 20: 1196-1203.

26. Jung JH, Chae YS, Moon JH, Kang BW, Kim JG, Sohn SK, Park JY, Lee MH, Park HY. TNF superfamily gene polymorphism as prognostic factor in early breast cancer. $\mathrm{J}$ Cancer Res Clin Oncol. 2010; 136: 685-694.
27. Azria D, Riou O, Castan F, Nguyen TD, Peignaux K, Lemanski C, Lagrange J-L, Kirova Y, Lartigau E, Belkacemi Y, Bourgier C, Rivera S, Noël G. Radiationinduced CD8 T-lymphocyte Apoptosis as a Predictor of Breast Fibrosis After Radiotherapy: Results of the Prospective Multicenter French Trial. EBioMedicine. 2015; 2: 1965-1973.

28. Balter S, Hopewell JW, Miller DL, Wagner LK, Zelefsky MJ. Fluoroscopically Guided Interventional Procedures: A Review of Radiation Effects on Patients' Skin and Hair 1. Radiology. 2010; 254: 326-341.

29. Kerns SL, de Ruysscher D, Andreassen CN, Azria D, Barnett GC, Chang-Claude J, Davidson S, Deasy JO, Dunning AM, Ostrer H, Rosenstein BS, West CML, Bentzen SM. STROGAR - STrengthening the Reporting Of Genetic Association studies in Radiogenomics. Radiother Oncol. 2014; 110: 182-188.

30. Kerns SL, Stock RG, Stone NN, Blacksburg SR, Rath L, Vega A, Fachal L, Gómez-Caamaño A, De Ruysscher D, Lammering G, Parliament M, Blackshaw M, Sia M, et al. Genome-wide association study identifies a region on chromosome 11q14.3 associated with late rectal bleeding following radiation therapy for prostate cancer. Radiother Oncol. 2013; 107: 372-376.

31. Schmitz A, Bayer J, Dechamps N, Thomas G. Intrinsic susceptibility to radiation-induced apoptosis of human lymphocyte subpopulations. Int J Radiat Oncol Biol Phys. 2003; 57: 769-778.

32. Laird NM, Horvath S, Xu X. Implementing a unified approach to family-based tests of association. Genet Epidemiol. 2000; 19 Suppl 1: S36-42.

33. Rabinowitz D, Laird N. A unified approach to adjusting association tests for population admixture with arbitrary pedigree structure and arbitrary missing marker information. Hum Hered. 2000; 50: 211-223.

34. Fisher RA. Statistical Methods for Research Workers. Gensis Publishing Pvt Ltd, editor. Edinburgh, London, Oliver and Boyd; 1925.

35. Rakovski CS, Xu X, Lazarus R, Blacker D, Laird NM. A new multimarker test for family-based association studies. Genet Epidemiol. 2007; 31: 9-17.

36. Chen H, Meigs JB, Dupuis J. Sequence kernel association test for quantitative traits in family samples. Genet Epidemiol. 2013; 37: 196-204.

37. Howie BN, Donnelly P, Marchini J. A flexible and accurate genotype imputation method for the next generation of genome-wide association studies. PLoS Genet. 2009; 5: e1000529.

38. Marchini J, Howie B, Myers S, McVean G, Donnelly P. A new multipoint method for genome-wide association studies by imputation of genotypes. Nat Genet. 2007; 39: 906-913. doi:10.1038/ng2088 